\title{
Electron Spin Resonance Studies of Anisotropy in Semiconducting Polymeric Films
}

\author{
DAG W. BREIBY, ${ }^{1 *}$ SHUICHI SATO, ${ }^{2}$ EMIL J. SAMUELSEN, ${ }^{1}$ KENJI MIZOGUCHI ${ }^{2}$ \\ ${ }^{1}$ Department of Physics, Norwegian University of Science and Technology, N-7491 Trondheim, Norway \\ 2 Department of Physics, Tokyo Metropolitan University, Hachi-oji, Tokyo, 192-0397 Japan
}

Received 28 March 2003; revised 26 June 2003; accepted 27 June 2003

\begin{abstract}
X-band electron spin resonance was employed to study the structural anisotropy in several polythiophene derivatives. Because of the dominating homogeneous width, the obtained absorption spectra were Lorentzian-shaped. Information about the structural anisotropy was obtained from the position and width of the absorption peak. Qualitatively, the anisotropy was in full agreement with earlier results from X-ray diffraction, including a flip in molecular orientation with respect to the film substrate between solution-cast and spin-cast films. With the Monte Carlo technique, the spectra were fitted with a biaxial $\mathbf{g}$ tensor, an anisotropy parameter $S$, and an intrinsic width $\sigma$. The simulations showed that $\mathbf{g}$ could be treated as pseudouniaxial, with the unique axis along the side chains rather than along the ring normal. Closed-form analytical expressions relating $\mathbf{g}$ to the anisotropy were obtained and used for a quantitative assessment of the molecular anisotropy. Because the molecular $\mathbf{g}$ tensor for these materials was not known, a known value of $S$ for one of the samples obtained by X-ray diffraction was used for normalization. Fairly consistent values were obtained for both $\mathbf{g}$ and $S$. ( 2003 Wiley Periodicals, Inc. J Polym Sci Part B: Polym Phys 41: 3011-3025, 2003
\end{abstract}

Key words: self-organization; ESR/EPR; polythiophene derivatives; conjugated polymers; Monte Carlo simulation

\section{INTRODUCTION}

Self-organization in soft materials has received much attention during the last decades, both theoretically and experimentally. ${ }^{1}$ The degree of structural order affects the properties of the materials directly. This is of major importance for materials considered for molecular electronics. Conjugated polymers are interesting in this context. Many of these polymers are semicrystalline

*Present address: Risø National Laboratory, P.O. Box 49, 4000 Roskilde, Denmark

Correspondence to: D. W. Breiby (E-mail: dag.werner. breiby@risoe.dk)

Journal of Polymer Science: Part B: Polymer Physics, Vol. 41, 3011-3025 (2003) (c) 2003 Wiley Periodicals, Inc. and exhibit preferred orientation. ${ }^{2}$ Several methods are known to enhance the tendency of ordering within the film plane, including stretching, (spin) casting of the material onto a thin layer of Teflon, and various poling techniques. ${ }^{2}$ For some conjugated polymers, a high degree of preferred orientation with the unique axis out of the film plane can be obtained..$^{3,4}$ Electron spin resonance (ESR) studies of the molecular orientation in conjugated polymers are discussed in this article.

The semicrystalline structures of several poly(alkylthiophene)s (PATs) and poly(3,4-dioxyethylenethiophene) (PEDOT) have been extensively studied previously, mainly with X-ray diffraction $(\mathrm{XRD})^{2-8}$ and recently also with scanning tunneling microscopy. ${ }^{9}$ These polymers consist of stiff 
planar molecules, with the thiophene rings and the side chains lying in the same plane. PATs have an orthorhombic structure, with $a$ chosen along the side chains, $b$ in the $\pi$-stacking direction, and $c$ along the polymer main chain. ${ }^{5}$ The crystalline regions are reported to display preferred orientation, depending on a number of external factors, particularly the solvent from which the film is cast. ${ }^{3,4}$ Remarkably, the crystalline $a$ axis (along the side chains) tends to orient perpendicularly to the substrate when the film is solution-cast, whereas when the film is spin cast, it sometimes prefers an orientation coplanar with the substrate. ${ }^{3,4}$ The stiff main polymer chains might be approximated to lie essentially flat in the film plane.

ESR has proven useful in the study of magnetic properties of conducting polymers, as it has contributed to establishing the existence of the rather exotic excitations found in these materials, namely, polarons. ${ }^{10,11}$ The theory of the anisotropy of the electronic $\mathbf{g}$ tensor due to the crystal field is well established. ${ }^{12,13}$ The literature is, however, scarce on examples of the use of ESR for studying preferred orientation in polymers. One exception is the study of mechanically stretched polyaniline by Wan and Wang, ${ }^{14}$ in which the focus is on the orientation of the main polymer chains along the stretch direction. Another is the work by Sato et al. ${ }^{15}$ on the out-of-plane orientation of polypyrrole, in which an oriented film was obtained by a novel technique employing a strong external magnetic field. A somewhat related discussion of obtaining orientational distribution functions (ODFs) of photooriented radical ions with ESR can be found in ref. 16 .

In a presentation at the ICSM 2002, Roth et al. ${ }^{17}$ have reported the values $\{2.00409,2.00332$, $2.00232\}$ for the $\mathbf{g}$ tensor of POT (defined in the next section), with a 2-mm wavelength. These powder sample values $g_{i i}$ are too high to be compatible with the observations to be presented here. Nevertheless, the quoted values are interesting because they indicate that the thiophene monomer cannot be taken as uniaxial despite its apparent ring symmetry. In this article, we present an anisotropy study by ESR and establish an analytical connection to the molecular ODF. The experimental spectra are simulated with the Monte Carlo technique. The discussion is given in light of earlier results obtained with XRD.

\section{EXPERIMENTAL}

\section{Samples: Chemistry and Film Preparation}

The materials studied included three different PATs with the alkyl groups of hexyl (PHT) and octyl (POT). The $\mathrm{FeCl}_{3}$-polymerized POT obtained from Neste OY was about $80 \%$ stereoregular and had a number-average molecular weight $\left(M_{\mathrm{n}}\right)$ of 21,500 and a weight-average molecular weight $\left(M_{\mathrm{w}}\right)$ of 43,800 . For PHT, both random regioregular (NR) and stereo regioregular (R) samples were studied. The NR sample, synthesized by O.R. Gautun at the Norwegian University of Science and Technology, had about $70 \%$ head-to-tail (h-t) couplings, $M_{\mathrm{n}}=26,800$, and $M_{\mathrm{w}}$ $=118,000$. The $\mathrm{R}$ sample purchased from Aldrich had approximately $98 \% \mathrm{~h}-\mathrm{t}$ couplings and $M_{\mathrm{w}}$ $\sim$ 87,000. PEDOT-toluene-sulfonic acid (TSA) cast on a solid polymeric substrate was also studied. The PEDOT-TSA was polymerized by the heating of the monomer and dopant in solution, and it probably had short chains ( $<20$ monomers). All the studied polymers had a flat, stiff, and bandlike conformation due to the conjugation.

Solution casting was used for obtaining thick (50-100- $\mu \mathrm{m})$ films, typically from chloroform solutions. For one of the samples (sample 4, cf. Table 1), the solvent was a mixture of tetrahydrofuran (THF) and chloroform, which is reported to give a higher degree of preferred orientation than either of the pure solvents. ${ }^{3,4}$ From a more diluted solution, some thinner films (a few micrometers thick) were also obtained and studied, notably for PEDOT-TSA and R-PHT. The main chains assumed a random orientation in the film plane. The side chains and the thiophene rings have been reported to preferentially orient perpendicular to the substrate for solution cast films. ${ }^{2}$

Films of POT were also prepared by spin casting from solutions with a concentration of approximately $10 \mathrm{mg} / \mathrm{mL}$ at about $1000 \mathrm{rpm}$ for a few seconds. This gave quite homogeneous thin films, about $200 \mathrm{~nm}$ thick, but with clearly visible stripes in the radial direction. Despite these thickness stripes, no preferred orientation of the main chains within the plane of spin-cast films has ever been reported. As mentioned in the introduction, the side chains have a tendency of lying flat on the substrate in the case of spin casting from nonregular materials. ${ }^{2,3}$ This implies that the thiophene rings are coplanar with the film plane.

A third way used to obtain films was the Langmuir technique of spreading small amounts of 
Table 1. Measured g-values

\begin{tabular}{rllcrr}
\hline No. & \multicolumn{1}{c}{ Sample } & \multicolumn{1}{c}{ Casting } & Thickness nm & $g_{\text {mean }}$ & $g_{\text {mod }} \times 10^{-5}$ \\
\hline 1 & POT & Spin $\perp$ & $200-300$ & 2.00215 & 5.4 \\
2 & POT & Langmuir & $10-20$ & 2.00228 & -8.1 \\
3 & POT & Spin $\|$ & $200-300$ & 2.00221 & 4.9 \\
4 & NR-PHT & Thick, solution-cast & $\sim 1.5 \times 10^{5}$ & 2.00213 & -49 \\
5 & R-PHT & Thick, solution-cast & $\sim 1.5 \times 10^{5}$ & 2.00211 & -19 \\
6 & NR-PHT & Langmuir & $10-20$ & 2.00215 & -17 \\
7 & R-PHT & Thin, solution-cast & $\sim 200$ & 2.00215 & -46 \\
8 & PEDOT-TSA & Thin, solution-cast & $\sim 200$ & 2.00296 & -7.6 \\
9 & PEDOT-TSA & Thin, solution-cast & $\sim 200$ & 2.00273 & -6.5 \\
\hline
\end{tabular}

a The parameters $g_{\text {mean }}$ and $g_{\text {mod }}$ are defined by eq 1 , both presumably having uncertainty in the last given digit. The PHT samples 4 and 5 were sufficiently thick that they could be released from the glass substrate; all other films were measured as deposited on their substrates. Just a few measurements were made for PEDOT, making $\mathbf{g}_{\text {mean }}$ and $\mathbf{g}_{\text {mod }}$ for samples 8 and 9 less precise than for the other samples. Sample 8 was doped with $\mathrm{PF}_{6}$ in addition to its TSA doping.

dissolved material on a water surface. A circular trough (diameter $=80 \mathrm{~mm}$, depth $=4 \mathrm{~mm}$ ) was filled with purified water. It was made of (hydrophobic) Teflon to obtain an upward meniscus of the water surface. One to two drops of a diluted PAT/chloroform solution, about $3 \mathrm{mg} / \mathrm{mL}$, were applied to the surface with a pipette. The resulting films were very thin, down to $10 \mathrm{~nm}$, yet continuous. Elsewhere, we have reported that such films show a high degree of preferred orientation of the crystalline $a$ axis (along the side chains, $a \sim 20.4 \AA$ for POT) perpendicular to the water subphase. The thickness, therefore, corresponds to about five molecular layers. Monolayers could not be obtained, probably because of the hydrophobicity of the side chains. ${ }^{18}$ By slowly draining the water out from the trough, the films were lowered down onto glass slides being submerged in the trough. More details on the properties of these films and the pseudo-LangmuirBlodgett technique can be found in ref. 18.

All the PAT films were doped by being dipped for a few seconds into an $\mathrm{NOPF}_{6}$ solution in acetonitrile $(\sim 40 \mathrm{mg} / \mathrm{mL})$. Acetonitrile is a selective solvent: it dissolved the salt, but not the polymer. The $\mathrm{PF}_{6}$ doped the film, which resulted in a color change from red to a faintly bluish luster. Especially for the thinnest samples, it was difficult to be sufficiently gentle to avoid damaging the film. Despite the $\mathrm{PF}_{6}^{-}$ions being quite heavy, dedoping took place. The doping level was, therefore, only estimated to about $25 \%$. When the sample tube was sealed, the doping was stable for (at least) several weeks. The $\mathrm{PF}_{6}^{-}$ions were superior to iodine ions in terms of both stability and a weak spin-orbit coupling, the latter being important to these ESR studies. PEDOT-TSA is stably doped as is, but sample 8 was accidentally also doped by $\mathrm{NOPF}_{6}$.

\section{ESR Studies}

The ESR measurements were carried out on a Japan Electron Optics Laboratory JES RE3X, operating at the X-band $(\sim 9.5 \mathrm{GHz})$ at the Tokyo Metropolitan University. The films were fit into quartz sample tubes with an internal diameter of approximately $4 \mathrm{~mm}$. For the ultrathin films sticking to a glass substrate, a small piece of the glass (with sample) was cut and inserted into the tube. For the thicker freestanding films, standard plastic foil was used to make a foil-sample-foil sandwich to fix the position of the sample inside the tube. The geometry is sketched in Figure 1. For the detection of anisotropy, the angle $\eta$ between the magnetic field and the sample film plane was varied stepwise.

For each selected value of $\eta$, the magnetic field was scanned through the magnetic resonances while the microwave frequency was kept essentially constant. The spectrometer measures the derivative of the absorption signal, which was recorded with a mechanical pen plotter and later digitized. When the derivative was zero, (absorption maximum), we made simultaneous readings of the magnetic field (using an ex situ NMR field meter) and the microwave frequency. The $\mathbf{g}$ value obtained from these measurements is called $\mathbf{g}_{\text {eff }}(\eta)$.

\section{RESULTS}

Typical ESR spectra obtained with the thiophenebased polymers are shown in Figure 2. The hori- 


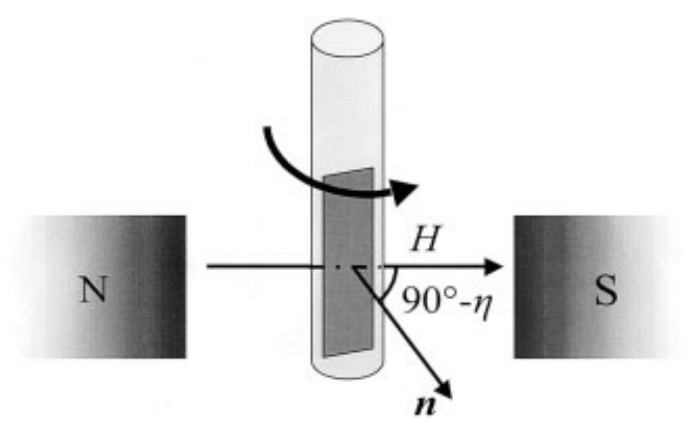

Figure 1. Sketch of the ESR measurement geometry depicting the magnet and the film with normal $\mathbf{n}$ inside the quartz glass sample tube. For clarity, the microwave cavity is not shown.

zontal peak-to-peak distance is denoted $H_{\mathrm{pp}}$, which is related to the half-width at half-maximum (hwhm), here denoted $H_{1 / 2}$, by $H_{\mathrm{pp}}=2 /$ $\sqrt{ } 3 H_{1 / 2}$. In the figure, spectra for R-PHT obtained for the angles $\eta=0^{\circ}$ and $90^{\circ}$ are shown. Both the resonance position $\mathbf{g}_{\text {eff }}$ and the line width $H_{\mathrm{pp}}$ are $\eta$-dependent. Only Lorentzian-shaped absorption curves were observed, with no finer details of the underlying structure. For this reason, the center of mass of the absorption curve coincides with the position of the maximum value; this is an impor-

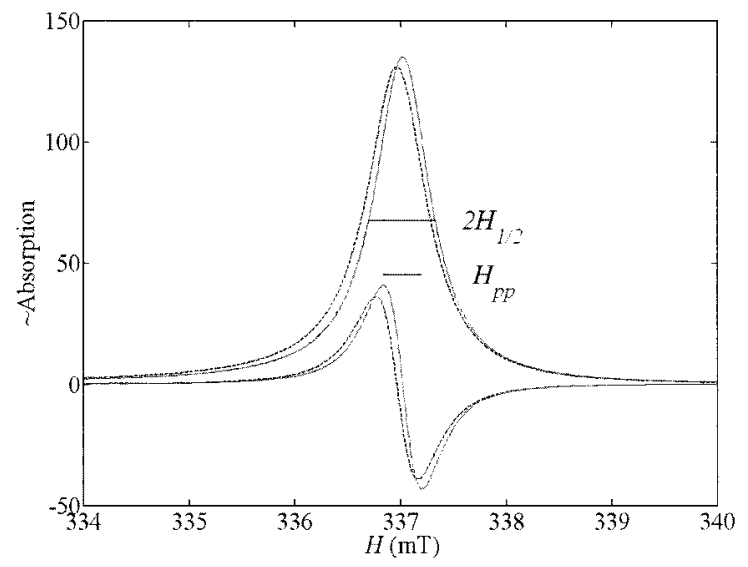

Figure 2. Typical ESR signals obtained for R-PHT sample 7 at $\eta=(-) 0^{\circ}$ and (-) $90^{\circ}$. Both the experimental derivative curves and the numerically integrated curves are shown. $H_{\mathrm{pp}}$ and the full width at half-maximum $\left(2 \mathrm{H}_{1 / 2}\right)$ of the integrated signal are indicated for the $\eta=0^{\circ}$ curve. At the point where the experimental lines cross the baseline, precise readings of both the magnetic field and the microwave frequency were made, giving the value of $\mathbf{g}_{\text {eff }}(\eta)$. Note the significant shift of the peak position toward lower $H$ with increasing $\eta$. The line width increases with $\eta$.

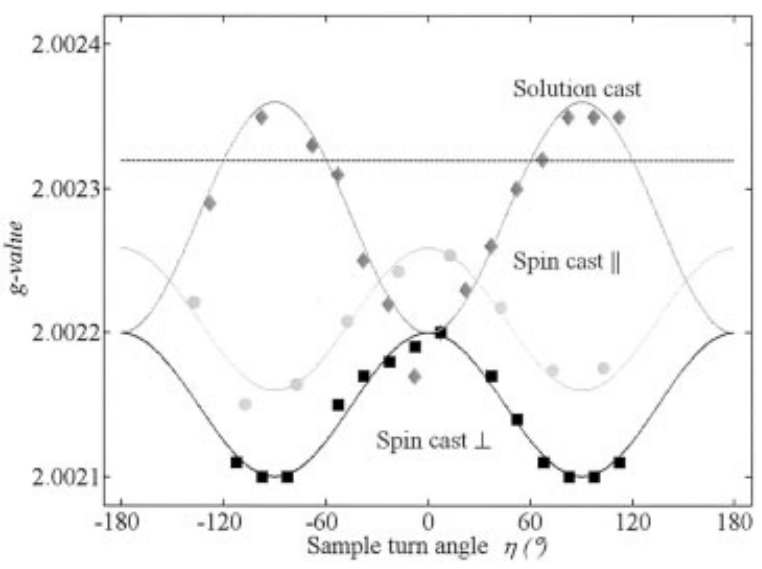

Figure 3. $g_{\text {eff }}(\eta)$ for solution-cast and spin-cast poly(octylthiophene). The symbols give the experimental data points, which are reasonably well fit by the sinusoidal curves. Note the effective phase flipping between the curves for solution casting versus spin casting. The horizontal, dashed line corresponds to $\mathbf{g}_{\mathrm{e}}$.

tant observation for the subsequent analysis in this work.

The experimental shifts in resonance position $g$ for all the studied films are collected in Table 1. The values were obtained by parameterization of the experimental $g$ values with

$$
g_{\text {eff }}=g_{\text {mean }}+g_{\text {mod }} \cos \left(2 \eta+\eta_{0}\right)
$$



Figure 4. $g_{\text {eff }}(\eta)$ for samples of $(-)$ partially stereoregular and (-) regioregular poly(hexylthiophene). The horizontal, dashed line represents $g_{\mathrm{e}}$. Two of the curves, for samples 4 and 7, have a very high amplitude $\left(g_{\text {mod }}\right)$ of the sinusoidal variation of $g$ with $\eta$. The other two curves, for samples 5 and 6 , have a more modest amplitude, with $g$ close to $g_{\mathrm{e}}$ for $\eta= \pm 90^{\circ}$. For one of the curves (sample 7), the raw data are also shown with symbols, being perfectly fitted by the sinusoidal curve. 


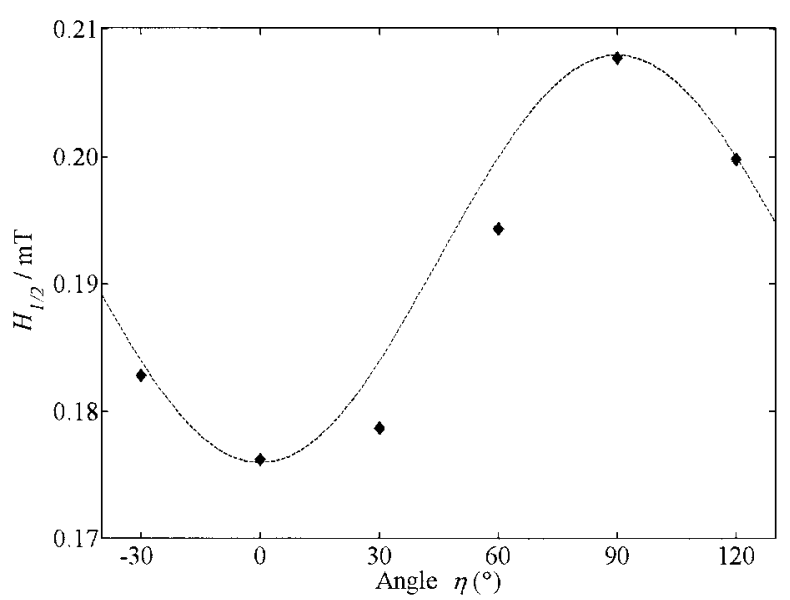

Figure 5. Observed line width as a function of $\eta$ for NR-PHT (sample 6). The dashed sinusoidal line is merely a guide to the eye.

where $g_{\text {mean }}$ is the average level of the experimental $g$ value as a function of $\eta, g_{\text {mod }}$ is the amplitude of the harmonic variation, and $\eta_{0}$ accounts for the arbitrary instrumental zero.

The results for the POT films are also shown in Figure 3. An important feature is the "phase flipping" between the solution-cast and spin-cast samples. The spin-cast film was cut into two different samples, for the radial thickness stripes orienting perpendicular $(\perp)$ and parallel $(\|)$ with respect to the sample strip length. In Figure 4, the data for the PHT films are plotted. In two cases, samples 4 and 7, a large anisotropy in $\mathbf{g}$ can be observed. This includes one sample with high stereoregularity and one with low stereoregularity. The thick regioregular PHT sample 5 was macroscopically rather crinkled and crispy, and this possibly explained its lower amplitude $g_{\text {mod }}$. An example of the variation in observed line width is given in Figure 5 for NR-PHT (sample 6). The (intrinsically doped) PEDOT-TSA data of Table 1 , for samples 8 and 9 , show a $g_{\text {mean }}$ value significantly higher than that for the other samples, whereas $\mathbf{g}_{\text {mod }}$ is comparable to that of the other materials.

\section{ANISOTROPY REVEALED BY ESR}

\section{Origin of the g-Shift and g-Anisotropy g-Value}

For an unpaired electron, the dimensionless $g$ value, or the electron Zeeman factor, is given by

$$
\nu=g H \frac{\beta_{\mathrm{e}}}{h}
$$

where $\nu$ is the Larmor frequency, $H$ is the magnetic field, $\beta_{\mathrm{e}}$ is the Bohr magneton, and $h$ is Planck's constant. The free-electron value $\mathbf{g}_{\mathrm{e}}$ is 2.00231930, for reference.

For radicals in a solid, the effective $g$-value is somewhat shifted from the $g_{\mathrm{e}}$ value because of interactions with the orbital electronic states. Anisotropy of the $g$-value means that different values for $g$ are obtained for different angles between the magnetic field and the molecular orientation. A detailed discussion and semiclassical calculation of the g-anisotropy for some organic molecules related to thiophene, such as benzene, can be found in ref. 12 . To calculate the $\mathbf{g}$ values $a$ priori is very demanding and is reported only for relatively simple molecules; one example is the recently published theoretical work on thiophene by Kleinschmidt et al. ${ }^{19}$

The generally accepted mechanism for charge transport in conjugated polymers involves polarons and bipolarons moving along and hopping between the individual main polymer chains. The polarons are free radicals observable by ESR, as they have unpaired electron spins. These elementary excitations are extended over a few rings, about seven or eight, along the main chain. ${ }^{10}$ The thiophene monomer consists of four carbon atoms and a sulfur atom, the latter being in some sense a stabilizing bridge in the conjugated structure. The delocalized $\pi$ orbitals originate from overlapping atomic $p_{z}$ orbitals. The highly anisotropic shape of the $\pi$ orbitals is presumably the dominant contribution to the g-anisotropy of the $\mathbf{g}$ factor through spin-orbit coupling.

In tensor notation, $\mathbf{g}$ is defined by ${ }^{13}$

$$
\hat{\mathbf{H}}=\beta_{\mathrm{e}}\left[H_{x} H_{y} H_{z}\right] \cdot\left[\begin{array}{ccc}
g_{x x} & 0 & 0 \\
0 & g_{y y} & 0 \\
0 & 0 & g_{z z}
\end{array}\right] \cdot\left[\begin{array}{c}
S_{x} \\
S_{y} \\
S_{z}
\end{array}\right]
$$

where $\hat{\mathbf{H}}$ denotes the spin Hamiltonian and $S$ is the spin operator. Throughout, nuclear interactions are neglected. With the molecular axis defined according to the Gordy convention ${ }^{12}$ as in Figure 6, the observed $g$ is given by

$$
\begin{array}{r}
g^{2}(\Theta, \Phi)=g_{x x}^{2} \sin ^{2} \Theta \cos ^{2} \Phi+g_{y y}^{2} \sin ^{2} \Theta \sin ^{2} \Phi \\
+g_{z z}^{2} \cos ^{2} \Theta
\end{array}
$$




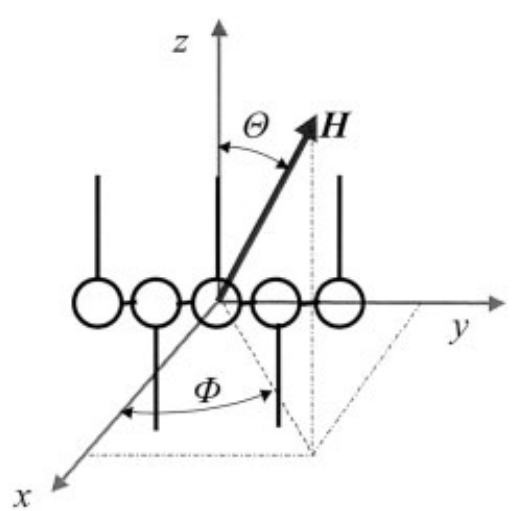

Figure 6. Sketch of a segment (five monomers shown) of a PAT polymer chain with the coordinate system $\{x, y, z\}$ for one of the monomers. The side chains, shown as idealized straight lines, are directed along the $z$ direction; the polymer main chain is in the $y$ direction; and the thiophene ring normal is directed along $x$. The angles $\Theta$ and $\Phi$ give the orientation of the external field $\mathbf{H}$ with respect to the monomer.

where the angles $\Theta$ and $\Phi$ give the direction of the magnetic field in the molecular frame $\{x, y, z\}$.

A sample containing molecules with different orientations yields a distribution of the resonance fields. We assume that every monomer independently obeys eq 4 . In the case of a highly anisotropic sample, particularly a single crystal, the individual contributions add up "in phase," which gives a measured absorption spectrum close to that of a single molecule (intermolecular interactions being neglected). Conversely, in the case of a less oriented sample, the contributions tend to smear the spectrum thereby reducing the amplitude $g_{\text {mod }}$ of eq 1 . For an isotropic orientation of the radicals, no dependence on the sample orientation will be observed; the observed characteristic powder spectrum is independent of the sample rotation angle $\eta$ defined in Figure 1 , and $g_{\text {mod }}$ is zero.

\section{Line Widths}

The observed spectrum is the convolution of the homogeneous and inhomogeneous broadening of the absorption lines. The inhomogeneous broadening is caused by the anisotropy, as discussed. Spin-lattice relaxation and motionally narrowed dipole-dipole interactions are the origin of the homogeneous (intrinsic) width. The relative widths of the contributions to the broadening are important. With an increasing magnetic field (and microwave frequency), the separation be- tween the resonances at $g_{i i}$ increases proportionally. Because the homogeneous width is generally independent or weakly dependent on $\mathbf{H}$, the resolution is increased.

In a recent work on polypyrrole performed at $20 \mathrm{GHz}$ (K-band), the homogeneous broadening was sufficiently small $(\sim 0.02 \mathrm{mT})$ that the distribution of resonance positions could be discerned. ${ }^{15}$ After deconvolution, the obtained spectra exhibited an angle-dependent (crystalline) part superposed on an angle-independent powder spectrum presumably originating from amorphous regions of the sample.

The situation is rather different in this study because the homogeneous broadening $(\sim 0.15-0.30$ $\mathrm{mT}$ ) gives rise to a low resolution. As shown in Figure 2, the experimental data exhibit only a Lorentzian. For this reason, only the average resonance position $g_{\text {eff }}$ and the line width $H_{\mathrm{pp}}$ are obtained.

\section{Integrated Intensities}

The integrated intensity, or photon absorption, can be obtained by two numeric integrations of the (differential) experimental curve. The absorption is linearly proportional to the number of unpaired spins. Because of the very different film thicknesses (cf. Table 1), the absorption varied by orders of magnitude between the samples.

\section{Quantifying the Anisotropy}

As mentioned previously, it is known from XRD that PAT films have uniaxial anisotropy. ${ }^{4}$ The main chains have a random distribution within the film plane, and the crystalline $a$ axis has a tendency of orienting with respect to the film normal. A general uniaxial ODF $f(\chi)$ for the unique axis is now discussed, cf. Figure 7. Throughout, $\{x, y, z\}_{i}$ is the coordinate system of an individual monomer, whereas the primed system $\left\{x^{\prime}, y^{\prime}, z^{\prime}\right\}$ is for the sample, the film normal being directed along $z^{\prime}$. The angle $\chi_{i}$ is defined as the angle between the unique axis (side chain) of a monomer and the film normal, that is, between $z$ and $z^{\prime}$. Because of the mirror plane symmetry, $f(\chi)$ is periodic in $\chi$ with a period of $180^{\circ}$.

The most frequently used parameter for giving a gross measure of uniaxial anisotropy is the socalled Hermans' parameter $S$ :

$$
S=\frac{1}{2}\left(3\left\langle\cos ^{2} \chi\right\rangle-1\right)
$$


The brackets indicate that an average is taken over the unit sphere. $S=0$ corresponds to an isotropic distribution, and $S=1$ corresponds to perfect polar orientation $\left(\chi=0^{\circ}\right)$, that is, orientation parallel to the film normal. Conversely, orientation in the film plane $\left(\chi=90^{\circ}\right)$ gives an $S$ value approaching -0.5 . Other values of $S(\in$ -0.5 and 1) give intermediate anisotropy, with $S$ $>0$ and $S<0$ implying a tendency of orienting the side chains perpendicularly and parallel to the film plane, respectively.

The Hermans' parameter is the first anisotropic term in a Legendre series expansion of the distribution function. For many spectroscopic techniques, it is not possible to measure the anisotropy beyond the second-order term $S$. XRD has the virtue of being capable of determining the ODF in full details, that is, the detailed parameterization of $f(\chi)$. For the thinnest films studied here, which cannot be lifted from the substrate, mostly qualitative X-ray observations exist for the anisotropy. ${ }^{3,18}$ In a quantitative XRD study of the anisotropy in thicker PAT films, ${ }^{4}$ the ODF of the crystallites was found to be well represented by a Gaussian-like function of the following form:

$$
f(\chi) \sim \exp \left(-B \sin ^{2} \chi\right)
$$

With increasing (positive) $B$, the distribution develops peaks in the polar regions, modeling an increased tendency of the side chains to align in the surface normal direction. Conversely, with increasingly negative values of $B$, a peak forms in the equatorial regions $\left(\chi \sim 90^{\circ}\right)$; modelling that, the side chains tend to be parallel to the film plane. For moderate anisotropy, $B$ is proportional to $S .{ }^{4}$

\section{Uniaxial g Tensor}

In this section, the parameter $g_{\text {eff }}(\eta)$, being the center of mass value of the absorption spectrum, is calculated analytically for a uniaxial system. A connection is established between the observed variation of $g_{\text {eff }}$, the sample rotation angle $\eta$, and the ODF. The inversion problem of obtaining information about the ODF from the variation of $g_{\text {eff }}$ is not straightforward. It is, not possible without further information to state whether a large $\mathbf{g}_{\text {mod }}$ value stems from a large orientational anisotropy or a big intrinsic difference between $g_{\perp}$ and $g_{\|}$.

The analysis presented here is adequate for systems in which the homogeneous line width is sufficiently large to dominate the inhomogeneous structure. In such cases, the center of mass value is close to the absorption maximum, that is, the measured value $g_{\text {eff }}$ (cf. Fig. 2).

For planar ring molecules, the approximation of isotropy of the g-value (cf. Figure 6) within the ring plane can sometimes be assumed, that is, $g_{z z}$ $\approx g_{y y}$ (cf. Figure 6 ). For molecules having a rotation axis of order 3 or higher, such an assumption is exact. For thiophene rings, because sulfur electronically differs significantly from the carbon atoms, this approximation is not necessarily valid. To the contrary, it appears from the biaxial simulations that the $g_{x x}$ and $g_{y y}$ parameters are closer (discussed later). Such uniaxial situation is modeled here.

In the uniaxial case, the orientation of a single molecule with respect to the magnetic field is fully determined by the angle $\Theta$ of Figure 6 . Therefore, uniaxiality gives

$g^{2}(\Theta)=g_{\perp}^{2} \sin ^{2} \Theta+g_{\|}^{2} \cos ^{2} \Theta=g_{\perp}^{2}+\left(g_{\|}^{2}-g_{\perp}^{2}\right) \cos ^{2} \Theta$

For the calculation of the observable effective $\mathbf{g}$ factor $\mathbf{g}_{\text {eff }}(\eta)$ as a function of the angle $\eta$ between the magnetic field and the film plane, $\eta$ must be related to the angles $\chi$ and $\phi$ describing the individual molecular orientation, cf. Figure 7. The angles $\chi$ and $\phi$ are related to the molecular anisotropy axis through

$$
\cos \Theta=\cos \varphi \sin \chi \cos \eta+\cos \chi \sin \eta
$$

Averaging over the normalized distribution function $f(\chi)$ gives

$$
g_{\text {eff }}(\eta)=\int_{0}^{2 \pi} \int_{0}^{\pi} g[\Theta(\eta, \varphi, \chi)] f(\chi) \sin \chi d \chi d \varphi
$$

The expression for the square of $g(\Theta)$ is given in eq 7 , and the factor $\sin (\chi)$ is the Jacobian in spherical coordinates. It is convenient to define a parameter $\Delta$ by

$$
\Delta \equiv g_{\|}^{2}-g_{\perp}^{2} \approx 2 g_{\perp}\left(g_{\|}-g_{\perp}\right)
$$

Because $\Delta$ is small, eq 7 can be approximated by 


$$
g=g_{\perp}\left(1+\frac{\Delta \cos ^{2} \Theta}{g_{\perp}^{2}}\right)^{1 / 2} \approx g_{\perp}+\left(g_{\|}-g_{\perp}\right) \cos ^{2} \Theta
$$

Inserting $\cos \Theta$ from eq 8 and averaging over the distribution function as indicated in eq 9 , we obtain the following expression:

$$
\begin{aligned}
\mathbf{g}_{\mathrm{eff}}(\eta)=\mathbf{g}_{\perp}+\frac{\Delta}{2 \mathbf{g}_{\perp}} & \left(\frac{1}{2}\left(1-3\left\langle\cos ^{2} \chi\right\rangle\right) \cos ^{2} \eta+\left\langle\cos ^{2} \chi\right\rangle\right. \\
& \left.+\frac{1}{2}\langle\cos \varphi \sin 2 \chi\rangle \sin 2 \eta\right)
\end{aligned}
$$

Because of the $\cos \varphi$ factor and the $\varphi$ independence of the ODF, the last term in eq 12 is identically zero. With $S$ of eq 5 substituted for $\left\langle\cos ^{2} \chi\right\rangle$, $g_{\text {eff }}(\eta)$ becomes

$$
\begin{array}{r}
g_{\text {eff }}(\eta)=g_{\perp}+\frac{\Delta}{2 g_{\perp}}\left(\frac{2 S+1}{3}-S \cos ^{2} \eta\right) \\
=g_{\perp}+\frac{\Delta}{g_{\perp}} \frac{S+2}{12}-\frac{S \Delta}{4 g_{\perp}} \cos 2 \eta=g_{\text {mean }} \\
+g_{\text {mod }} \cos 2 \eta
\end{array}
$$

Eq. 13 gives theoretical expressions for the parameters $\mathbf{g}_{\text {mean }}$ and $\mathbf{g}_{\text {mod }}$ of eq 1, both being functions of $S$ and $\Delta$ :

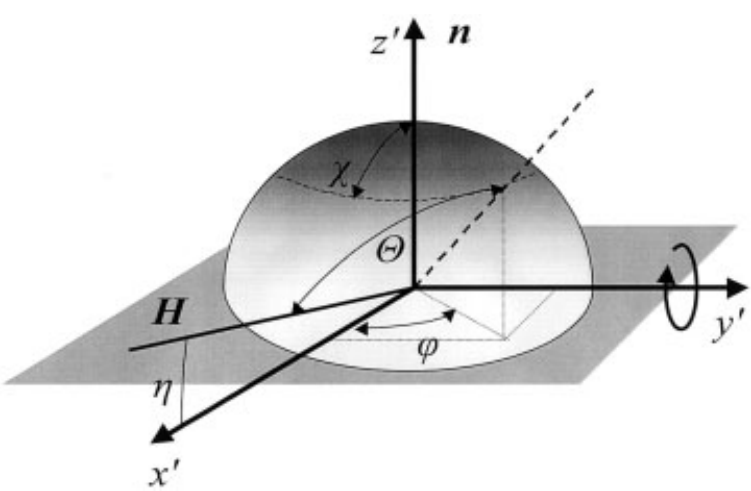

Figure 7. Sketch of the relationships between the various angles for the uniaxial geometry. The magnetic field $\mathbf{H}$ forms an angle $\eta$ with the film $\left(x^{\prime}-y^{\prime}\right)$ plane, chosen by the rotation of the sample about the $y^{\prime}$ axis. The shading of the semisphere illustrates a uniaxial distribution. The angle $\Theta$ is the angle between $\mathbf{H}$ and a unique molecular axis (a monomer side chain) with the orientation $\left(\varphi_{i}, \chi_{i}\right)$. The vector $\mathbf{n}$ denotes the unique axis of the film normal.

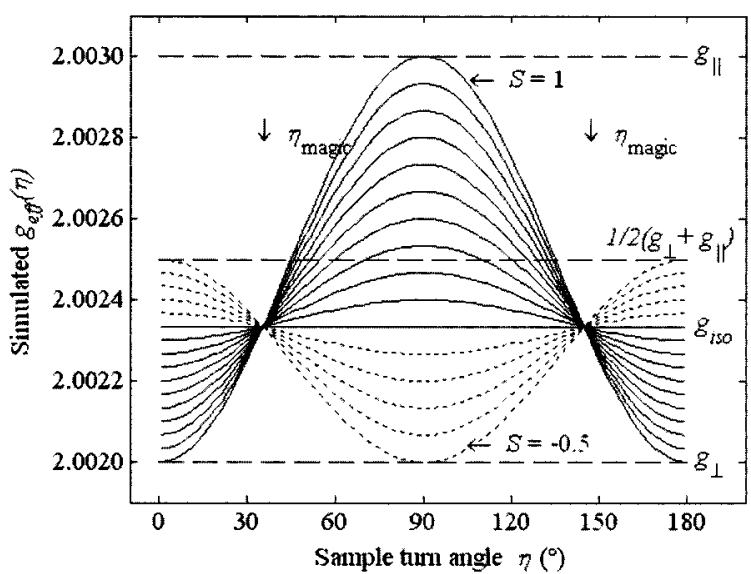

Figure 8. Calculated values of $g_{\text {eff }}(\eta)\left(g_{\perp}=2.0020\right.$ and $g_{\|}=2.0030$ ) for the parameter $S$ with values of $\{-0.5,-0.4, \ldots, 1\}$. The curves for negative $S$ values are given with dotted lines, having as extrema $\mathbf{g}_{\perp}$ and $0.5\left(\mathbf{g}_{\perp}+\mathbf{g}_{\|}\right)$. For positive $S$ values, the limiting values are $\mathbf{g}_{\perp}$ and $\mathbf{g}_{\|}$. The three horizontal, dashed lines indicate the different limiting values. At the angular values marked $\eta_{\text {magic }}$, all curves cross the horizontal line $g_{\text {iso }}$, corresponding to an isotropic orientation of the monomers. See the text for further discussion.

$$
\begin{aligned}
\mathbf{g}_{\text {mean }}=\mathbf{g}_{\perp}+\frac{\Delta(S+2)}{12 \mathbf{g}_{\perp}} \approx \mathbf{g}_{\perp}+ & \frac{1}{6}\left(\mathbf{g}_{\|}-\mathbf{g}_{\perp}\right)(S+2) \\
& =\mathbf{g}_{\text {iso }}-\frac{1}{3} \mathbf{g}_{\text {mod }}
\end{aligned}
$$

$$
\mathbf{g}_{\text {mod }}=-\frac{S \Delta}{4 \mathbf{g}_{\perp}} \approx-\frac{1}{2}\left(\mathbf{g}_{\|}-\mathbf{g}_{\perp}\right) S
$$

As required, the value of $\mathbf{g}$ for an isotropic distribution $(S=0), \mathbf{g}_{\text {iso }}$, is $\eta$-independent:

$$
\mathbf{g}_{\text {iso }}=\mathbf{g}_{\perp}+\frac{\Delta}{6 \mathbf{g}_{\perp}} \approx \frac{1}{3}\left(2 \mathbf{g}_{\perp}+\mathbf{g}_{\|}\right)
$$

This is actually the trace of the $\mathbf{g}$ tensor. By inserting $\cos 2 \eta=1 / 3$, corresponding to an angle $\eta_{\text {magic }}$ of $35.3^{\circ}$, it is seen from eq 13 that the value $g_{\text {iso }}$ is obtained regardless of the anisotropy $S$.

Calculated $g$-values as a function of $\eta$ obtained from eq 13 are plotted in Figure 8. For films with $S>0$ (solution cast, side chains tending to be parallel to the film normal), the external magnetic field $\mathbf{H}$ is along the molecular $z$ axis for $\eta$ $=90^{\circ}$. This implies a $g$-value approaching $\mathbf{g}_{\|}$of a single thiophene ring as $S$ tends to unity. For $\mathbf{H}$ parallel to the substrate $\left(\eta=0^{\circ}\right), g_{\text {eff }}$ approaches $g_{\perp}$ for $S$ near unity. 
Conversely, for films (spin-cast in this study) with $S<0$ and $\mathbf{H}$ parallel to the film normal, $g_{\text {eff }}$ approaches $\mathbf{g}_{\perp}$ as $S$ approaches -0.5 . For $\mathbf{H}$ parallel to the substrate, the measured $\mathbf{g}$ value is given by the average $0.5\left(\mathbf{g}_{\perp}+\mathbf{g}_{\|}\right)$because of the isotropic orientation within the film plane. These limiting values of $g_{\text {eff }}$ are readily obtained from eq 13 and are indicated with dashed lines in Figure 8.

For reference, we note that if the unique axis is along the hetero ring normal rather than the side chain, the analysis must be slightly modified, as the ODF for the ring normal is different from (yet related to) the $\operatorname{ODF} f(\chi)$ for the side chains. ${ }^{4}$ It can be shown rigorously that $S$ must then be replaced by $S_{\mathrm{u}}=-0.5 S .^{20}$

\section{Biaxial g Tensor}

With a biaxial $\mathbf{g}$ tensor, the full orientation of the individual monomer must be specified. This can be done, for example, with the so-called $x y z$ convention of Euler angles. ${ }^{21}$ With the molecular axes $\{x, y, z\}$ defined as in Figure 6 , the molecular orientation in the sample frame $\left\{x^{\prime}, y^{\prime}, z^{\prime}\right\}$ is then defined by a yaw angle $\varphi$ about the $z^{\prime}$ axis, followed by a pitch $\theta$ about an intermediary $y^{\prime}$ axis and a roll $\psi$ about the final $x^{\prime}$ axis. In our case, the roll $\psi$ is, therefore, about the thiophene ring normal (cf. Fig. 6). The magnetic field forms an angle $\eta$ with the film $\left(x^{\prime}-y^{\prime}\right)$ plane: $\mathbf{H}=\langle\cos \eta, 0$, sin $\eta\rangle$. From the angles $\{\varphi, \theta, \psi, \eta\}$, the angles $\Phi$ and $\Theta$, relating the magnetic field to the molecular frame, are found to be

$$
\begin{aligned}
& \Phi(\varphi, \theta, \psi, \eta) \\
& \quad=\operatorname{atan}\left(\begin{array}{r}
\cos \eta(\sin \psi \sin \theta \cos \varphi-\cos \psi \sin \varphi) \\
+\sin \eta \cos \theta \sin \psi \\
\cos \eta \cos \theta \cos \varphi-\sin \eta \sin \theta
\end{array}\right)
\end{aligned}
$$

$\Theta(\varphi, \theta, \psi, \eta)=\operatorname{acos}[\cos \eta(\cos \psi \sin \theta \cos \varphi$

$$
+\sin \psi \sin \varphi)+\sin \eta \cos \theta \cos \psi]
$$

The angle $\chi$ between the molecular $z$ axis and the film normal $\mathbf{n}$ parallel to $z^{\prime}$ is obtained by

$$
\chi=\operatorname{acos}(\cos \theta \cos \psi)
$$

Considerations similar to those for the uniaxial case can be applied to the biaxial case. $g_{\text {eff }}$ is then given by

$$
g_{\text {eff }}=g_{x x}+\Delta_{y}\left\langle\sin ^{2} \Theta \sin ^{2} \Phi\right\rangle+\Delta_{z}\left\langle\cos ^{2} \Theta\right\rangle
$$

where $\Delta_{y} \equiv g_{y y}-g_{x x}$ and $\Delta_{z} \equiv g_{z z}-g_{x x}$, both small compared to the $g_{\text {ii }}$ values. A tedious calculation of the averages in eq 20 , in which the variable $\phi$ has been integrated out, gives

$$
\begin{aligned}
&\left\langle\sin ^{2} \Theta \sin ^{2} \Phi\right\rangle=\frac{1}{2}\left(3\left\langle\cos ^{2} \theta \cos ^{2} \psi\right\rangle+1-3\left\langle\cos ^{2} \theta\right\rangle\right) \\
& \times \cos ^{2} \eta-\left\langle\cos ^{2} \theta \cos ^{2} \psi\right\rangle+\left\langle\cos ^{2} \theta\right\rangle \\
&\left\langle\cos ^{2} \Theta\right\rangle= \frac{1}{2}\left(1-3\left\langle\cos ^{2} \theta \cos ^{2} \psi\right\rangle\right) \cos ^{2} \eta \\
&+\left\langle\cos ^{2} \theta \cos ^{2} \psi\right\rangle
\end{aligned}
$$

Because of the explicit expectation value $\left\langle\cos ^{2} \theta\right\rangle$ in eq 21 , it is evidently not quite as simple a dependence on $S$ as for the uniaxial case. However, $g_{\text {eff }}(\eta)$ is proportional to $\cos ^{2} \eta$ also in the general biaxial case, as shown by eqs 20-22. In the case of uniaxiality, $g_{\mathrm{xx}}=g_{\mathrm{yy}}, \Delta_{\mathrm{y}}$ vanishes and eq 13 from the uniaxial considerations is retrieved.

To further investigate the implications of eqs $20-22$, the ODF must be specified. It is already assumed that the ODF is symmetric with respect to $\varphi$. Two ODFs are of particular interest. Firstly, letting $\psi$ and $(\sin \theta+1) / 2$ be uniformly distributed on $[0,2 \pi)$ and $[0,1)$, respectively, gives a perfectly isotropic "powder" distribution.

Secondy, as mentioned in the introduction, a realistic model, particularly for the thin films, is that the main chains are essentially parallel to the film plane. From the definition of the angles $\varphi$, $\theta$, and $\psi$, it is seen that $\psi \approx 0^{\circ}$ implies a horizontal $y$ axis; that is, the main chains are in the film plane. Because of the cos dependence on $\psi$ in eqs 21 and $22, \psi \approx 0^{\circ}$ allows some $10^{\circ}$ buckling of the main chains to a good approximation. Assuming that the ODF is essentially a Dirac $\delta$ function $\delta(\psi)$ for the angle $\psi$, yields an angle $\chi$ (eq 19) now equal to $\theta$, and eqs 21 and 22 are considerably simplified to

$$
\begin{gathered}
\left\langle\sin ^{2} \Theta \sin ^{2} \Phi\right\rangle=\frac{1}{2} \cos ^{2} \eta \\
\left\langle\cos ^{2} \Theta\right\rangle=\frac{1}{2}\left(1-3\left\langle\cos ^{2} \theta\right\rangle\right) \cos ^{2} \eta+\left\langle\cos ^{2} \theta\right\rangle
\end{gathered}
$$

Plugging these expressions into eq 20 and substituting in $S$, we obtain the following expression: 


$$
\begin{aligned}
g_{\text {eff }}=\left(g_{x x}+\frac{1}{4} \Delta_{y}+\Delta_{z}\right. & \left.\frac{1}{6}(S+2)\right) \\
& +\frac{1}{4}\left(\Delta_{y}-2 S \Delta_{z}\right) \cos 2 \eta
\end{aligned}
$$

This is the biaxial (with $\psi=0^{\circ}$ ) equivalent to eq 13 , giving predictions for $g_{\text {mean }}$ and $g_{\text {mod }}$ of eq 1 . It is effectively a modification of the uniaxial distribution and reduces to eq 13 for $\Delta_{y}=0$. One important difference between eqs 13 and 25 is that a $\cos (2 \eta)$ dependence is predicted even for $S=0$, reflecting the anisotropy imposed by $\psi$ being set to 0 .

\section{Monte Carlo Simulations}

To model the absorption spectrum is a problem well suited for Monte Carlo simulations. To this end, the form of the distribution function $f(\chi)$ was assumed to be the one obtained previously by $\mathrm{XRD}^{4}$ (cf. eq 6). Throughout, the homogeneous broadening $\sigma$ was assumed to be independent of orientation. Typically, $10^{4}$ monomers were simulated, being sufficient for reasonable reproducibility. With a lower value of $\sigma$ than in this case, more monomers are probably necessary to obtain smooth curves.

The absorption spectrum for given values of $\eta$ and $B$ was simulated by the following algorithm. For all $\left(\sim 10^{4}\right)$ molecules (monomers),

- Let the orientation of the $i$ th molecule be given by (Random main chain orientation for $S=0$ )

$$
\begin{aligned}
& \varphi_{i}=\operatorname{rand}_{1} \cdot 2 \pi \\
& \theta_{i}=\operatorname{asin}\left(2 \cdot \operatorname{rand}_{2}-1\right) \\
& \psi_{i}=\operatorname{rand}_{3} \cdot 2 \pi
\end{aligned}
$$

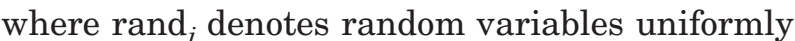
distributed on $[0,1]$. The asin function is invoked to account for the Jacobian in spherical coordinates.

- Calculate $\Phi_{i}, \Theta_{i}$, and $\chi_{i}$ with eqs 17,18 , and 19 , respectively.

- Calculate $g_{i}=g_{i}\left(\Phi_{i}, \Theta_{i}\right)$ with eq 4.

- Give molecule $i$ a weight according to eq 6 :

$$
W_{i}=\exp \left(-B \sin ^{2} \chi_{i}\right)
$$

- Add the contribution from this $i$ th monomer to the simulated spectrum with a Lorentzian with amplitude $W_{i}$ and line width $\sigma$

Then, calculate the anisotropy $S$ from the $\chi_{i}$ values. If desired, quantities such as the spectrum maximum, the line width, and the center of mass can be obtained from the simulated spectrum. The absorption curve can be differentiated numerically for comparisons with the experimental data.

The method (eqs 26-28) for picking values of $\phi_{i}, \theta_{i}$, and $\psi_{i}$ is unbiased in the sense that for $B$ $=0, S$ tends to 0 , that is, full isotropy. Modeling a powder, the simulated spectrum is invariant under interchanging $g_{i i}$ values, as it should be. Equivalently, the powder spectra are $\eta$-independent.

A choice of angles corresponding to having the main chains essentially in the film plane is given by the following:

('Main chains orienting in the plane for all $S$ ')

$$
\begin{aligned}
& \varphi_{i}=\operatorname{rand}_{1} \cdot 2 \pi \\
& \theta_{i}=\frac{\pi}{2}-\operatorname{asin}\left(2 \cdot \operatorname{rand}_{2}-1\right) \\
& \psi_{i}=0
\end{aligned}
$$

As described, this parameter choice will force the main chains ( $y$ direction) to be aligned with the sample plane, and this implies an anisotropy seen in the simulations as an $\eta$ dependence also for the $S=0$ case. The definition of the $\theta_{\mathrm{i}}$ is modified with respect to eq 27 to ensure that $S$ goes to $\mathrm{O}$ as $B$ approaches zero.

The two models are equivalent in the limit of high anisotropy. It is, of course, possible to construct more refined models for the distribution of the angles $\theta$ and $\psi$ with more complicated functions.

The same algorithms can be used to simulate uniaxial spectra. Because the weighing gives preference to the $z$ axis, the most straightforward implementation is to always take $g_{\|}$in the $z$ direction.

\section{FITTING OF DATA}

The experimental data are now related to the theoretical framework developed in the previous 
section. The appropriate values for $g_{i i}$ are not known, either for PATs or for PEDOT, and the system is, therefore, underdetermined. For the uniaxial algebraic treatment of $\mathbf{g}_{\text {eff }}(\eta)$, the three parameters $\mathbf{g}_{\perp}, \mathbf{g}_{\|}$, and $S$ appear in the expressions for the two experimental variables $\mathbf{g}_{\text {mean }}$ and $\mathbf{g}_{\bmod }$. For the biaxial simulations, the experimental line width $H_{\mathrm{pp}}$ is taken into account, but the parameter $\sigma$ and the full biaxial $\mathbf{g}$ tensor enter the description. In both cases, different sets of parameter values are, therefore, able to fit the data. Because the system is underdetermined, we did not attempt to apply the analytical biaxial analysis. As shown algebraically, the parameters $S$ and the intrinsic molecular $g$ anisotropy (i.e., $\Delta_{y}$ and $\Delta_{z}$, eq 20) are strongly correlated. They are also closely connected to the homogeneous line width $\sigma$. Absolute bounds for the orientational anisotropy $S$ and for $g_{i i}$ can be established by a consideration of certain limiting cases. Clearly, $S$ must be within -0.5 to 1 , and the $g_{i i}$ values must be sufficiently close not to imply an absorption peak broader than what is observed experimentally.

From the well-established facts concerning the molecular orientation in solution-cast and spincast samples, it is evident that for solution-cast samples, $g_{\text {eff }}$ must shift toward $g_{z z}$ as $\eta$ increases to $90^{\circ}$. Similar considerations apply to the spincast sample having the side chains (and the main chains) in the film plane, with the ring normals ( $x$ axis) serving as the unique axis. Therefore, for $\eta$ approaching $90^{\circ}, g_{\text {eff }}$ will be approaching $g_{x x}$. However, as evident from the subsequent discussion, the values of $g_{x x}$ and $g_{y y}$ are too close to be reliably discerned. In accordance with the common practice in reporting powder $\mathbf{g}$ values, $g_{x x}$ is here taken as the lowest of the two values differing from $g_{z z}$.

As mentioned, changing to a higher value of $\mathbf{H}$ increases the resolution, and this would probably allow the simultaneous measurement of both the g tensor and the anisotropy. To this end, work is in progress. ${ }^{22}$ However, with anisotropy data from XRD as additional input, a quantitative treatment of the ESR data normalized to an absolute scale can be performed. Importantly, the same highly anisotropic solution-cast NR-PHT sample reported in Table 1 (sample 4) has also been studied by XRD, although in the undoped state. ${ }^{4}$ The obtained XRD value for Hermans' parameter, $S=0.45 \pm 0.05$, can be used as a first estimate of the anisotropy measured by ESR. Typical XRD values for NR-POT give $S$ values between 0.05 and 0.25 for moderately anisotropic solution-cast samples. ${ }^{4}$ For ultrathin films sticking to substrates, only qualitative XRD data exist. $^{2,3,18}$

Fundamental differences in the nature of ESR and XRD make the anisotropy as probed by the respective techniques in some sense complementary. Whereas XRD probes only the (periodic) crystalline regions of the polymers, the ESR signal has contributions from all regions of the material. Because the amorphous state has a more random orientation than the crystalline regions, it is reasonable to assume that the measured degree of preferred orientation is higher with XRD than with ESR. Observations by XRD indicate that in highly oriented samples, even the noncrystalline regions also exhibit a tendency of preferred orientation, ${ }^{4}$ suggesting that the difference between the ESR and XRD anisotropy might be least pronounced for such samples. ESR requires unpaired electron spins, and studies of conjugated polymers are therefore usually done in the doped state, or the sample is excited with light. XRD can also be performed on doped material, but it is more often done with undoped samples. We assume that the doping level has a negligible effect on the degree of orientation.

\section{Simulations of the ESR Spectra}

With Monte Carlo simulations as outlined, good fits of the experimental spectra were obtained. Examples are given in Figure 9 for the highly anisotropic R-PHT sample 7 and in Figure 10 for the much less anisotropic POT sample 2. The amplitudes of the individual curves contain no vital information and are chosen to increase the readability of the figures.

\section{Poly(hexylthiophene)}

The PHT data of samples 4 and 7 were important for the analysis presented here. These samples exhibited a sufficiently high anisotropy that the value of $g_{z z}(\approx 2.0030)$ and the mean value $(\approx 2.0015)$ for $g_{x x}$ and $g_{y y}$ were obtained with a comparably low uncertainty. To this end, the constraints from the X-ray studies that the molecular $z$ direction should be predominantly along the sample normal and that the anisotropy be about $S$ $\sim 0.45$ were imposed. Interestingly, the simulations were relatively insensitive to the deviation of $g_{x x}$ and $g_{y y}$ from their mean value. The example shown in Figure 9 is for $g_{x x}=2.0013$ and $g_{y y}$ 
$=2.0017$, yielding a marginally better fit than $g_{x x}$

$=g_{y y}=2.0015$.

No difference was found between the highly stereoregular and less stereoregular samples, as can be concluded directly from Figure 4 .

\section{Poly(octylthiophene)}

As XRD data were not available for any of the POT samples, it was assumed that the parameters $g_{i i}$ and $\sigma$ were reasonably similar to those obtained for PHT. An additional objective was that these parameters should yield good fits for both the solution-cast and spin-cast samples. With the $\mathbf{g}$ tensor and $\sigma$ as given in Table 2, the spectra for both samples 2 and 3 were reproduced, including the reversed shift with $\eta$ seen for sample 3. As for the PHT samples, the main dependence of $g_{x x}$ and $g_{y y}$ is on their mean value.

\section{PEDOT-TSA}

Because of the small number of observations, the results for the PEDOT-TSA sample are more uncertain than for the two other materials. Nevertheless, the obtained values for $g_{i i}$ and $S$ are in reasonable agreement with the $\mathrm{PHT}$ and POT values.

\section{Discussion}

With the anisotropy for the PHT samples chosen to be equal to that observed by XRD, reasonable, yet possibly somewhat low, anisotropy values were obtained for the other samples. This might


Figure 9. ESR spectra for R-PHT, sample 7. The solid lines were obtained with $g_{x x}=2.0013, g_{y y}=$ 2.0017 , and $g_{z z}=2.0030$ combined with an anisotropy parameter $B=3$ giving $S \approx 0.44$. Further discussion can be found in the text.
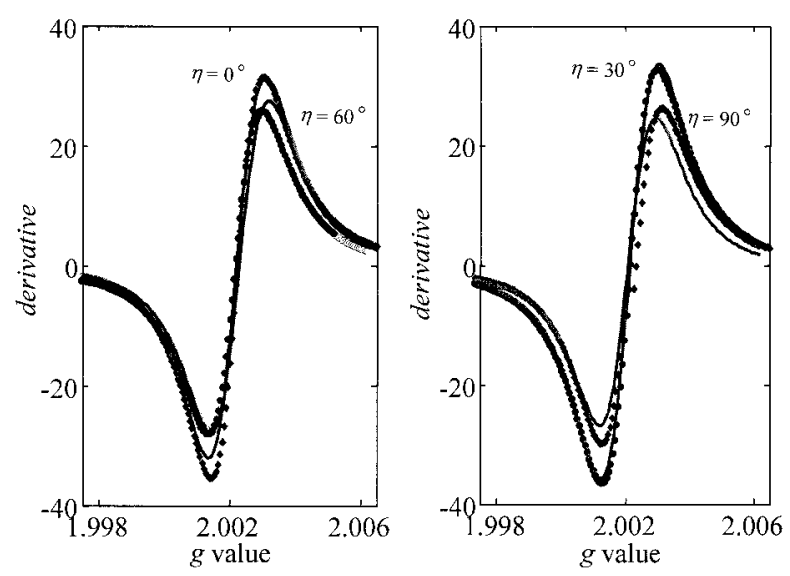

Figure 10. ESR spectra for Langmuir cast POT, sample 2 . The solid lines were obtained with $g_{x x}=2.0018$, $g_{y y}=2.0021$, and $g_{z z}=2.0030$ combined with an anisotropy parameter $B=0.6$ giving $S \approx 0.09$. Further discussion can be found in the text.

enforce the observations in ref. 4 (on the quantification of anisotropy) that for the most highly oriented samples, the difference between the crystalline and amorphous regions is not pronounced.

The obtained values for the anisotropy are in fair agreement with reported X-ray data for both the POT and PHT samples. ${ }^{4}$ As reported earli$\mathrm{er}^{2-4}$ the sample cast from a THF/chloroform mixture (sample 4) exhibited a particularly high anisotropy. Also, the thin solution-cast R-PHT sample 7 had an almost identical anisotropy (cf. Fig. 4). The error in the values given for $g_{i i}$ is about \pm 0.0002 for the PHT samples and higher for POT and PEDOT-TSA. The uncertainty in $S$ is estimated to about $20 \%$ on an absolute scale. For relative comparisons between the samples (especially of the same material), the uncertainty is much lower.

The deviation in the line width between the experimental and fitted curves for $\eta=60^{\circ}$ in Figure 9 might reflect either that the choice of parameters is nonoptimal or that the model lacks some features. Suggestions for extending the description include modifying the single homogeneous line width $\sigma$ to be dependent on the angles $\Theta$ and $\Phi$. Another extension would be to modify the ODF (eq 6) as outlined in the Monte Carlo section. In fact, the alternative model with $\psi=0^{\circ}$ was also tested, but the results were found to be largely equivalent.

Interestingly, it is evident from the fitted spectra that the $\eta$ dependence of the line width $H_{\mathrm{pp}}$ is largely reproduced. The experimental data are 
Table 2. Fitted Values of the $\mathbf{g}$ Tensor, the Intrinsic Line Width $\sigma$ (given as hwhm), and the Anisotropy in Terms of $S$ for Most of the Samples

\begin{tabular}{|c|c|c|c|c|c|c|c|}
\hline No. & Sample & Preparation & $g_{x x}\left(\sim \mathbf{g} \perp_{\text {low }}\right)$ & $g_{y y}\left(\sim \mathbf{g} \perp_{\text {high }}\right)$ & $g_{z z}\left(\sim \mathbf{g}_{\|}\right)$ & $\sigma(\mathrm{mT})$ & $S$ \\
\hline 1 & POT & Spin $\perp$ & & & & & \\
\hline 2 & POT & Langmuir & 2.0018 & 2.0021 & 2.0030 & 0.22 & 0.09 \\
\hline 3 & POT & Spin $\|$ & 2.0018 & 2.0021 & 2.0030 & 0.22 & -0.10 \\
\hline 4 & NR-PHT & Thick, solution-cast & 2.0013 & 2.0017 & 2.0029 & 0.16 & 0.44 \\
\hline 5 & R-PHT & Thick, solution-cast & 2.0015 & 2.0020 & 2.0029 & 0.15 & 0.22 \\
\hline 6 & NR-PHT & Langmuir & & & & & \\
\hline 7 & R-PHT & Thin, solution-cast & 2.0013 & 2.0017 & 2.0030 & 0.16 & 0.42 \\
\hline 8 & PEDOT-TSA & Thin, solution-cast & & & & & \\
\hline 9 & PEDOT-TSA & Thin, solution-cast & 2.0021 & 2.0025 & 2.0034 & 0.30 & 0.07 \\
\hline
\end{tabular}

modelled to good accuracy on the basis of the molecular $\mathbf{g}$ tensor, the line width $\sigma$ and the anisotropy $S$. Also the intrinsic widths $\sigma$ appears to be material specific, cf. Table 2 .

Concluding, the main insights obtained from simulating the spectra are that the system is approximately uniaxial with the molecular $z$-axis as a unique axis, and that the $\eta$-dependence of the experimental line width is largely explained. That the molecular $z$-axis is an approximate unique axis is the basis for the next section.

\section{Obtaining the Orientational Order Parameter from the Analytical Expressions}

With the result from the simulations that the materials had an approximate unique axis along $z$, it was interesting to see whether similar results could be obtained within the analytical uniaxial approximation. As mentioned previously, because the expressions for the two quantities $g_{\text {mean }}$ (eq 14) and $g_{\text {mod }}$ (eq 15) involve three unknowns $\left(g_{\perp}\right.$, $g_{\|}$, and $S$ ), input from XRD was used to estimate the $\mathbf{g}$ and $S$ values on an absolute scale.

One might guess that a possible uniaxiality of the $\mathbf{g}$ tensor would exist with the ring normal as the unique axis, but that is not the case, as also observed by Monte Carlo simulations (not shown). To reproduce the $\eta$-dependence of $g_{\text {eff }}$ while maintaining a Lorentzian shaped absorption spectrum, a huge intrinsic width $\sigma$ would be required, yielding an absorption peak much wider than what is observed experimentally.

As described, the Monte Carlo studies show that to a reasonable approximation, the values of $g_{x x}$ and $g_{y y}$ are sufficiently close that the molecular $z$ direction can be considered a unique axis. Solving eqs 14 and 15 with the experimental val- ues for $g_{\text {mean }}$ and $g_{\text {mod }}$, with $S=0.50$, we obtained 2.0013 and 2.0033 for $\mathbf{g}_{\perp}$ and $\mathbf{g}_{\|}$, respectively. Assuming that all samples have the same value for $\Delta$ (eq 10), we can estimate the molecular $\mathbf{g}$ values and the anisotropy $S$ for all the samples. A certain agreement is found between this easy calculation and the more elaborate Monte Carlo approach. The obtained values are listed in Table 3, which should be compared with Table 2 .

A particularly interesting feature of Table 3 is that this procedure is consistent in the sense that the $\mathbf{g}$ values for the PHT, POT, and PEDOT-TSA materials are well grouped, increasing in the order PHT $<$ POT $<$ PEDOT-TSA while their molecular $g$ anisotropy remains constant ( $\Delta$ is assumed to be fixed). For the POT samples, no significant difference in $\mathbf{g}$ is found between the spincast samples oriented with the radial spin direction perpendicular (sample 1) and parallel (sample 2) to the sample rotation axis.

In conclusion, the results obtained by the two methods are in fair agreement. The analytical expressions serve well in finding first estimates for the $g$ values and the anisotropy, which can be used also as initial values for the biaxial simulations.

\section{CONCLUSIONS}

ESR was applied for studying the anisotropy of some thiophene-based, doped conjugated polymers (PHT, POT, and PEDOT-TSA) in the form of solution-cast and spin-cast films and as Langmuir films formed on water surfaces and transferred to glass.

Because of the broad homogeneous width, the finer absorption features were smeared to give 
Table 3. Obtained Values of $S, g \perp$, and $g_{\|}$for the Various Samples with the Uniaxial Approximation of $g_{x x} \approx g_{y y}{ }^{a}$

\begin{tabular}{|c|c|c|c|c|c|c|c|c|}
\hline No. & Sample & Preparation & $g_{\text {mean }}$ & $g_{\text {mod }} \times 10^{-5}$ & $S_{\text {ref }}$ & $g \perp$ & $g_{\|}$ & $S$ \\
\hline 1 & POT & Spin $\perp$ & 2.00215 & 5.4 & & 2.0015 & 2.0035 & -0.06 \\
\hline 2 & POT & Langmuir & 2.00228 & -8.1 & & 2.0016 & 2.0036 & 0.08 \\
\hline 3 & POT & Spin \| & 2.00221 & 4.9 & & 2.0016 & 2.0035 & -0.05 \\
\hline 4 & $N R-P H T$ & Thick, solution-cast & 2.00213 & -49 & 0.5 & 2.0013 & 2.0033 & \\
\hline $5^{\mathrm{b}}$ & R-PHT & Thick, solution-cast & 2.00211 & -19 & & 2.0014 & 2.0034 & 0.19 \\
\hline 6 & NR-PHT & Langmuir & 2.00215 & -17 & & 2.0014 & 2.0034 & 0.17 \\
\hline 7 & R-PHT & Thin, solution-cast & 2.00215 & -46 & & 2.0013 & 2.0033 & 0.47 \\
\hline 8 & PEDOT-TSA & Thin, solution-cast & 2.00296 & -7.6 & & 2.0023 & 2.0042 & 0.08 \\
\hline 9 & PEDOT-TSA & Thin, solution-cast & 2.00273 & -6.5 & & 2.0021 & 2.0040 & 0.07 \\
\hline
\end{tabular}

a The columns $g_{\text {mean }}$ and $g_{\text {mod }}$ have been copied from Table $1 . S_{\text {ref }}$ denotes the value obtained by XRD, which allowed the estimation of $\mathbf{g} \perp$ and $\mathbf{g}_{\|}$for sample 4 , the reference sample. This gave a value of $\Delta=0.008$. For the remaining samples, the listed values for $\mathbf{g} \perp$ and $\mathbf{g}_{\|}$were calculated under the assumption that $\Delta$ could be taken as a constant. The column $S$ gives Hermans' parameter (eq 5), which, together with $\mathbf{g} \perp$ and $\mathbf{g}_{\|}$, reproduces the values for $\mathbf{g}_{\operatorname{mean}}$ and $\mathbf{g}_{\bmod }$ via eqs 14 and 15 .

$\mathrm{b}$ This film was rather crinkled, and this probably contributed to its low value of $\mathbf{g}_{\bmod }$.

only a Lorentzian-shaped absorption signal with width $H_{\mathrm{pp}}$ centered about $g_{\text {eff. }}$ Orientational anisotropy revealed itself as a $\cos (2 \eta)$ dependence of $g_{\text {eff }}$ on the angle $\eta$ between the film surface and the applied magnetic field. The amplitude of this variation could be used as a measure of the degree of preferred orientation, as expressed by the Hermans' parameter $S$. The normalization of the amplitude scale was achieved via combination with $S$ values from XRD data available for one of the samples. For this purpose, it was conjectured that the molecular $\mathbf{g}$ value anisotropy of the thiophene ring was only weakly dependent on the type of side chain and was, therefore, similar in PHT, POT, and PEDOT.

The data fitting was performed by way of Monte Carlo simulations, involving a three-component (orthorhombic) $\mathbf{g}$ tensor, a uniaxial orientational distribution function, and a single constant $\sigma$ governing the homogeneous broadening. The simulations reproduced the main features of the experimental spectra, namely $g_{\text {eff }}(\eta)$ and $H_{\mathrm{pp}}(\eta)$. The materials were found to be approximately uniaxial, with the unique axis directed along the side chains.

By exploiting the fact that the center of mass of the absorption spectrum coincided approximately with the absorption maximum because of the large intrinsic width, we obtained analytical expressions for the observed $g_{\text {eff }}$ values as a function of the monomer $\mathbf{g}$ tensor and $S$. For uniaxial symmetry, particularly simple expressions were obtained, which were used in an approximate analysis. The $g_{\text {ii }}$ values obtained were fairly consistent.
The anisotropy parameters obtained show that the degree of anisotropy depends on the solvent used in preparing the films. A mixture of chloroform and THF gave rise to much higher anisotropy than pure chloroform, in agreement with previously reported X-ray results. Solution-cast films and pseudo-Langmuir-Blodgett films exhibited a preferred orientation of the polymer side chains normal to the film surface. The spin-cast films, however, showed a preference for the side chains parallel to the film surface. No particular effect was seen of the degree of stereoregularity of the polymers.

This work demonstrates that the ESR technique can be an appropriate method for studying the anisotropy of conjugated polymer films. It is well suited for comparative studies between various samples. If the molecular $\mathbf{g}$ tensor or (as in this study) the anisotropy is known, quantitative work normalized to an absolute scale can be performed.

The authors thank Hirokazu Sakamoto at the Tokyo Metropolitan University for valuable discussions. D. W. Breiby gratefully acknowledges the Norway-Japan Sasakawa Foundation for financial funding and the ESR group at Tokyo Metropolitan University for its hospitality during his stay in Japan. The Norwegian Research Council is gratefully acknowledged for D. W. Breiby's Ph.D. grant.

\section{REFERENCES AND NOTES}

1. Semiconducting Polymers: Chemistry, Physics and Engineering; Hadziioannou, G.; van Hutten, P. F., Eds.; Wiley: Weinheim, 2000. 
2. Samuelsen, E. J.; Aasmundtveit, K. E.; Breiby, D. W. In Electronic and Optical Properties of Conjugated Molecular Systems in Condensed Phases; Hotta, S., Ed.; Research Signpost: in press.

3. Aasmundtveit, K. E.; Samuelsen, E. J.; Guldstein, M.; Steinsland, C.; Flornes, O.; Fagermo, C.; Seeberg, T. M.; Pettersson, L. A. A.; Inganäs, O.; Feidenhans'l, R.; Ferrer, S. Macromolecules 2000, 33, 3120.

4. Breiby, D. W.; Samuelsen, E. J. J Polym Sci Part B: Polym Phys 2003, accepted for publication.

5. Samuelsen, E. J.; Mårdalen, J. In Handbook of Organic Conductive Molecules and Polymers; Nalwa, H. S., Ed.; Wiley: New York, 1997; Vol. 3, p 87.

6. Breiby, D. W.; Samuelsen, E. J.; Groenendaal, L. B.; Struth, B. J Polym Sci Part B: Polym Phys 2003, 41, 945.

7. Sirringhaus, H.; Brown, P. J.; Friend, R. H.; Nielsen, M. M.; Bechgaard, K.; Langeveld-Voss, B. M. W.; Spiering, A. J. H.; Janssen, R. A. J.; Meijer, E. W.; Herwig, P.; de Leeuw, D. M. Nature 1999, 401, 685.

8. Yamamoto, T.; Kokubo, H.; Morikita, T. J Polym Sci Part B: Polym Phys 2001, 39, 1713.

9. Grévin, B.; Rannou, P.; Payerne, R.; Pron, A.; Travers, J. P. J Chem Phys 2003, 118, 7097.
10. Lu, Y. Solitons and Polarons in Conducting Polymers; World Scientific: River Edge, NJ, 1988.

11. Conductive Polymers: Spectroscopy and Physical Properties; Mizoguchi, K.; Kuroda, S.; Nalwa, H. S., Eds.; Wiley: New York, 197; Vol. 3, p 251.

12. Gordy, W. Theory and Applications of Electron Spin Resonance; Wiley: New York, 1980.

13. Weil, J. A.; Bolton, J. R.; Wertz, J. E. Electron Paramagnetic Resonance, Wiley: New York, 1994.

14. Wan, M. X.; Wang, J. Synth Met 1997, 84, 751.

15. Sato, S.; Sakamoto, H.; Mizoguchi, K.; Morioka, H.; Kimura, T. Synth Met 2003, 135-136, 421.

16. Vorob'ev, A. K.; Ferster, S.; Gurman, V. S. Russ J Phys Chem 2000, 74, 1867.

17. Roth, H.-K.; Krinichnyi, V. I. Synth Met 2003, 137, 1431.

18. Breiby, D. W.; Samuelsen, E. J.; Konovalov, O.; Struth, B. Submitted for publication.

19. Kleinschmidt, M.; Tatchen, J.; Marian, C. M. J Comput Chem 2002, 23, 824.

20. Lovell, R.; Mitchell, G. R. Acta Crystallogr Sect A 1981, 37, 135.

21. Goldstein, H. Classical Mechanics; Addison-Wesley: Reading, MA, 1980.

22. Breiby, D. W.; Sato, S.; Mizoguchi, K.; Samuelsen, E. J. To be submitted for publication, 2003. 